\title{
A Vibrotactile Alarm System for Pleasant Awakening
}

Korres, Georgios; Jensen, Camilla Birgitte Falk; Park, Wanjoo; Bartsch, Carsten; Eid, Mohamad

Published in:

IEEE Transactions on Haptics

Link to article, DOI:

10.1109/TOH.2018.2804952

Publication date:

2018

Document Version

Peer reviewed version

Link back to DTU Orbit

Citation (APA):

Korres, G., Jensen, C. B. F., Park, W., Bartsch, C., \& Eid, M. (2018). A Vibrotactile Alarm System for Pleasant Awakening. IEEE Transactions on Haptics, $11(3), 357$ - 366. https://doi.org/10.1109/TOH.2018.2804952

\section{General rights}

Copyright and moral rights for the publications made accessible in the public portal are retained by the authors and/or other copyright owners and it is a condition of accessing publications that users recognise and abide by the legal requirements associated with these rights.

- Users may download and print one copy of any publication from the public portal for the purpose of private study or research.

- You may not further distribute the material or use it for any profit-making activity or commercial gain

- You may freely distribute the URL identifying the publication in the public portal

If you believe that this document breaches copyright please contact us providing details, and we will remove access to the work immediately and investigate your claim. 


\title{
A Vibrotactile Alarm System for Pleasant Awakening
}

\author{
Georgios Korres, Camilla Birgitte Falk Jensen, Wanjoo Park, Carsten Bartsch and Mohamad Eid
}

\begin{abstract}
There has been a vast development of personal informatics devices combining sleep monitoring with alarm systems, in order to find an optimal time to awaken a sleeping person in a pleasant way. Most of these systems implement auditory feedback as the alarm signal, which is not always pleasant and may disturb other sleepers in the same space. In this paper, we present an adaptive alarm system that detects sleeping cycles and triggers the alarm signal during shallow sleep, in order to minimize sleep inertia. Since tactile sensation is associated with positive valence, vibrotactile stimulation is investigated as a silent alarm to enhance pleasant awakening. Three modulation techniques to render the tactile stimuli for pleasant awakening are considered, namely simultaneous, continuous, and successive stimulation. Two experimental studied are conducted. Experiment 1 studied exogenous attention towards tactile stimulation in a multimodal scenario (involving visual and haptic interactions) with fully awake individuals. Results from the attention task and the subjective valence rating suggest that the vibrotactile stimulation should be based on the continuous modulation, since this not only is very perceivable but also associated with positive attention. Experiment 2 evaluated the user experience with tactile stimulation patterns during sleep. Results confirmed the findings of experiment 1. Continuous modulation was rated highest for pleasant yet arousing sleep-awake transition.
\end{abstract}

Index Terms-Vibrotactile Stimulation, Affective Haptics, Evaluation/Methodology, Haptic I/O, Pervasive Computing, Health Care, Psychology, User-centered Design.

\section{INTRODUCTION}

W AKING up can sometimes be unpleasant. In many cases, being awoken by the alarm clock during a period of deep sleep causes a person to feel weak and/or confused, or experience a general fatigue during the day [1]. A potential solution to this problem involves determining the sleeping phase the slumberer is experiencing at the instant of supposed alarm-firing, and adapting the actual alarm firing instant accordingly. Preferably, the sleeper will be awakened during a period of light sleep. Another solution suggests using an arousing and/or pleasant stimulation. A combined approach, based on adaptive alarm clock and tactile-stimulation response, is what we are interested in exploring to enhance pleasant yet arousing awakening.

Adaptive alarm clock systems (both research efforts and commercial products) detect sleep cycles and fire an audio alarm during shallow sleep [1], [2], [3], [4], [5], [6]. In contrast to regular alarm clocks, an adaptive alarm clock chooses an optimal time to wake the user up using contextual knowledge (such as calendar information, sleep quality, and psychophysiology). For instance, HappyWakeUp [2] detects user movements in a bed using the microphone of the mobile phone to identify sleep stage (shallow sleep during the Rapid Eye Movement or REM stage and deep sleep during non-REM stage) and wakes the sleeper up during shallow sleep (REM stage). Sony has recently patented an alarm pillow with electrodes on the surface to come in

- G. Korres, W. Park, C. Bartsch, and M. Eid are with the Engineering Division, New York University Abu Dhabi, United Arab Emirates.

E-mail: see https://wp.nyu.edu/aimlab/

- C. Jensen is with the Technical University of Denmark, Denmark.

Manuscript received April 13, 2017. contact with the head to read brain wave signals [7]. The system analyzes the collected EEG signals to determine when the user goes into the REM or non-REM stages and turns on a buzzer attached to the pillow as soon as the person gets out of deep sleep.

Most alarm systems use auditory modality to display the alarm signal. A potential approach for reducing sleep inertia during wake up would be to apply appropriate tactile stimulation to draw the sleeper's attention (to help wake up) in a pleasant manner. A relation exists between tactile stimulation and drawing attention [8]. Attention can be divided into endogenous and exogenous: The endogenous attention describes the voluntary direction of attention to a particular point, such as paying attention to a person at a cocktail party. In contrast, exogenous or involuntary attention is the reflexive shifts of attention to unexpected or uninformative events, such as someone calling one's name at a cocktail party, or if a fly suddenly lands on one's arm [9].

The majority of attention research has focused on single sensory modalities, such as vision, audition, touch and even olfaction and gustation (visual [10] or haptics [11]). However, in everyday life, we commonly operate across different sensory modalities to facilitate the selection of relevant information. The classic cocktail party problem, where we direct our auditory attention to one particular voice in order to have a conversation in a noisy environment is actually misleading: we often rely on many other sensory modalities such as visual information from lip-movements, facial expressions, and gestures. In addition, we often ignore irrelevant sensory inputs, such as the feel of one's clothes (tactile), the smell of someone's perfume (olfactory), and perhaps even the taste of one's drink (gustatory). Never- 
theless, it is difficult to say what our attention is directed towards when we sleep, since this might depend on the sleep phase and the individual.

In this paper we take a novel approach for pleasant yet arousing awakening by utilizing tactile stimulation as an alarm response, and we validate this approach via two usability experiments. Hypothesizing that our attention is not necessarily directed towards tactile inputs, experiment 1 investigates measuring the exogenous attention towards different tactile stimuli in a multimodal scenario (visual and haptic). Experiment 2 evaluates the effects of sleeping on valence/arousal reactions by testing the alarm system with sleeping individuals. Both experiments confirmed that continuous tactile stimulation is the most pleasant (rated highest for valence reaction) yet effective in waking up (rated high for arousal which is highly correlated to shifting sleep to awake state [12]) .

The remainder of the paper is organized as follows: In section II we present the literature of tactile stimulation. Section III summarizes the software architecture and hardware implementation of the Aegis system (used in this study) and details the sleep stage extraction algorithm. Section IV introduces experiment 1 to evaluate exogenous attention towards different tactile stimuli in a multimodal scenario (including experimental setup, analysis, and results). In section $\mathrm{V}$, we present experiment 2 to evaluate tactile stimulation patterns with sleeping individuals. Finally, section VI summarizes the paper findings and provides perspectives for future work.

\section{Tactile Stimulation Literature}

The concept of tactile stimulation has been around for decades, since Geldard [13] in the 1950s studied the temporal and spatial aspects of tactile discrimination on skin and wrote: for some kinds of messages the skin offers a valuable supplement to ears and eyes. Early research on haptic stimulation has focused on applications aiding blind or visually impaired people [14], but later developments included entertainment and gaming [15], mobile and touchscreen interaction [16], emotional and interpersonal communication [17], [18], [19], and health care (such as physical rehabilitation [20]) [21].

Tactile stimulation can display natural, intuitive, and rich information by modulating the control signal for an array of low cost vibration motors. These modulations can be modified by changing the frequency, amplitude (intensity), Burst Duration (BD), Inter-Burst Interval (IBI), spatial distribution and direction, resulting in different haptic patterns. Many studies have compared the subjective ratings of different haptic patterns using the classic 9-point valence and arousal scale (commonly used to measure emotions [22]); a literature review on affective haptics is available here [23]. The valence level indicates how positive or negative a stimulus is, whereas the arousal level indicates how calming or exciting the stimulus is. An example of how emotions can be distributed into these two scales is shown in Russell's model [24] in Fig. 1. In addition, some studies compare different patterns on additional scales such as cognitive scales of continuity [25], smoothness [26], strength and rhythm [27], or speed [28].

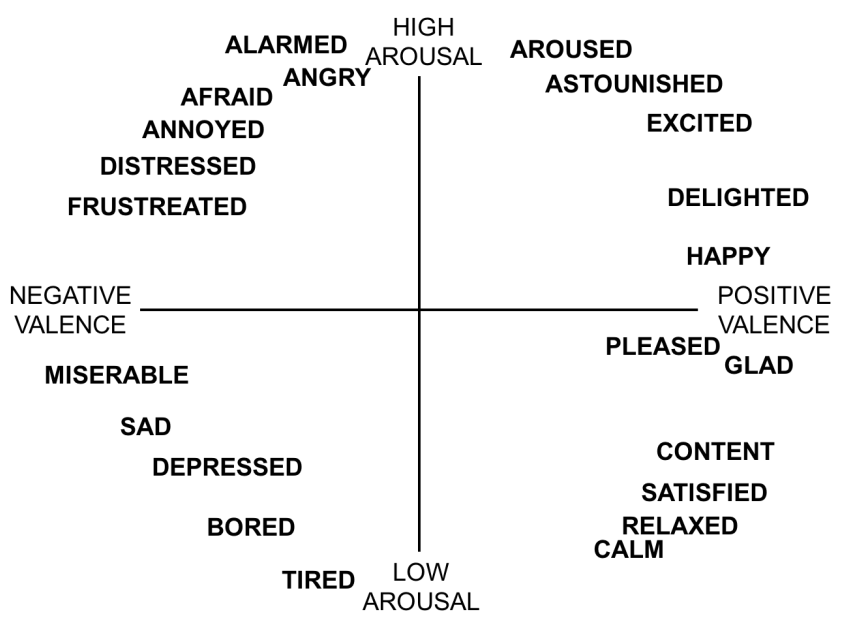

Fig. 1. Russell's model illustrates how the valence and arousal scale can be used to describe different emotions (adopted from [24]).

Due to the multiple possibilities for modulating haptic patterns, many studies choose to keep some parameters fixed, while concentrating on the effects of others, which often results in varying conclusions. However, many studies on affective haptics report continuous apparent motion being perceived as pleasant, whereas simultaneous stimuli were rated more unpleasant [29], [28]. Other studies focus more on how the different parameters contribute or affect the continuous feeling [15], [25], [30].

Raisamo et al. [29] found a correlation between continuous tactile stimulation and pleasant emotional reactions whereas simultaneous stimuli were rated more unpleasant. While others examined the effect of frequency, amplitude, duration, direction and body site on continuity and subjective preferences [15], [25], Rahal et al. showed an effect of gender, limb size and intensity [30]. Although most studies on affective haptics report preferences towards more continuous stimulation, this might not be the most suitable when used as an alarm clock (for sleeping individuals). In this case, a soothing continuous motion might not draw enough attention upon itself to cause the user to wake up. Instead it might become integrated into the users dream. On the other hand, using a very arousing and aggressive stimulation could result in an unpleasant awakening that might lead to a dislike of the product. Thus, we are interested in examining not only the subjective emotional ratings of haptic patterns but also how fast the subject's attention is shifted towards the different haptic patterns.

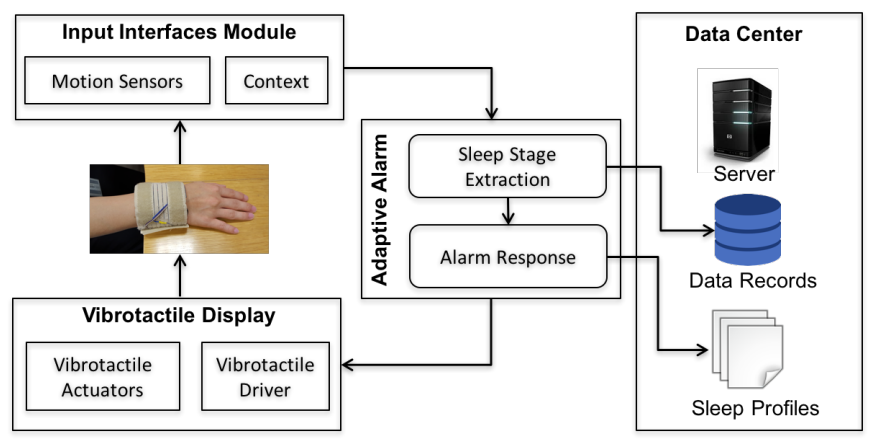

Fig. 2. Software architecture for the Aegis system. 


\section{AEGIS Adaptive Alarm Implementation}

The Aegis system design is shown in Fig. 2 (a comprehensive description and preliminary testing is published in our previous work [31]). In this section, we will focus on the development of the Adaptive Alarm Subsystem which is the heart of the Aegis system. It includes the Sleep Stage Extraction component and the Alarm Response component.

\subsection{Sleep Stage Extraction}

The Sleep Stage Extraction component analyzes body movement from the accelerometer to classify sleep stages into awake, REM and non-REM. Signal artifacts, caused mostly by sleeper movements that may severely deteriorate the accelerometer readings and thus the motion estimation, are removed via a preprocessing phase. The sleep stage extraction flowchart is shown in Fig. 3.

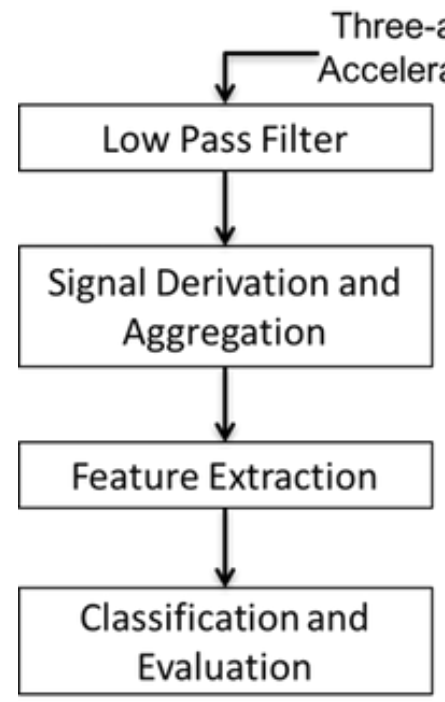

Fig. 3. Sleep stage extraction flowchart.

The preprocessing phase is adopted from the work presented in [32]. The raw three-axis acceleration data are converted first into the SI units $\left(\mathrm{m} / \mathrm{s}^{2}\right)$ by a calibration procedure. The three signals $\left(a_{x}, a_{y}, a_{z}\right)$ are then passed through the following phases:

1) Low Pass Filter: The raw three-axis acceleration data are passed through a second order low-pass filter with a cutoff frequency of $18 \mathrm{~Hz}$ to cancel out high frequency noise [33]. The transfer function of this filter is:

$$
H(z)=\frac{0.0625+0.125 z^{-1}+0.0625 z^{-2}}{1+1.3 z^{-1}-0.5 z^{-2}}
$$

2) Signal Derivation and Aggregation: A second order derivative operation is applied to each of the threeaxis acceleration data to remove baseline wander and gravity components. The transfer function for the derivative operation is shown in equation 2 . Next, the three axis-acceleration signals were combined into a single (axis-independent) signal by calculating the absolute sum.

$$
H(z)=\frac{1}{T^{2}}\left[1-z^{-1}\right]^{2}
$$

3) Feature Extraction: The single, axis-independent signal reduces computational effort in further processing steps and increase the amplitude resolution of the body movements. From now on, the algorithm is independent of the orientation of the accelerometer sensor at the beginning or during sleep. The data is divided into 30-second epochs. Feature extraction was based on the calculation of the body movement index [34]. For each 30-second epoch $e$, a body movement index was calculated using equation 3 . The body movement index is calculated as follows: two separate integration operations are applied to the axis-independent signal over a two second $\left(I_{2}\right)$ and four second $\left(I_{4}\right)$ window (the signals $I_{2}$ and $I_{4}$ have different response characteristics to different periods of movement activity). Digital integration was chosen as it is more suitable for identifying movement, compared to other methods such as zero crossing or time above threshold [35]. The body movement index is then calculated as the summation of the series of $I_{2}$ and $I_{4}$ values starting from the 4th second till the 30th second, in a step size of 2 seconds. The average over a window of 18 subsequent epochs of the body movement index is also used as another feature for classification.

$$
M(e)=\sum_{e}\left(\frac{I_{2}}{I_{4}}\right)^{2}
$$

4) Classification and Evaluation: Naive Bayes classifiers [36] are used to distinguish awake, REM and non-REM sleep using the body movement index feature. Comparative evaluation was performed by recording data with the SOMNOwatch plus EEG 6 device [37]. Two healthy subjects (one male and one female) were recorded during seven consecutive nights. The Aegis system was placed on the subjects arm, while reference hypnograms were collected using the SOMNOwatch plus EEG 6 device.

The reference data is also divided into 30-second epochs from the accelerometer signals, and was preprocessed with the DOMINOlight software by SOMNOmedics $\mathrm{GmbH}$. The body movement index feature was used together with the ground truth information recorded from the SOMNOwatch $^{\mathrm{TM}}$ plus EEG 6 device to train the Naive Bayes classifiers extract sleep stage (awake, REM and nonREM). A snapshot comparing results we got using the Aegis system and the SOMNOwatch ${ }^{\mathrm{TM}}$ plus EEG 6 device for detecting awake, REM and non-REM phases over 8 hours of sleep is shown in Fig. 4.

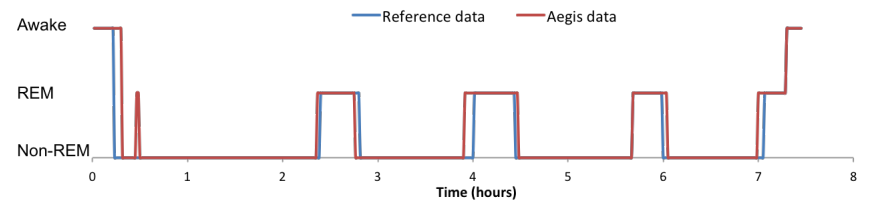

Fig. 4. Example hypnogram with the different sleep stages (Awake, REM and non-REM) over around 8 hours of sleep. 
A total of 12550 epochs was collected from two subjects and used for training and evaluation, $18.2 \%$ of those epochs were REM-epochs. Results for classification of REM, nonREM, and Awake phases are given in Table 1 for each subject and over a course of seven days. The classifier has an average accuracy of $94.24 \%$ compared to the $97.79 \%$ average accuracy for the reference SOMNOwatch ${ }^{\mathrm{TM}}$ plus EEG 6 device.

TABLE 1

Classification accuracy (awake, rem and non-rem)

\begin{tabular}{lllll}
\hline \multirow{2}{*}{ Days } & \multicolumn{2}{l}{ Subject 1 (male) } & \multicolumn{2}{l}{ Subject 2 (female) } \\
\cline { 2 - 5 } & Error & Sleep Time & Error & Sleep Time \\
\hline 1 & $6.06 \%$ & $7.45 \mathrm{hrs}$ & $6.48 \%$ & $7.90 \mathrm{hrs}$ \\
2 & $5.69 \%$ & $8.03 \mathrm{hrs}$ & $6.21 \%$ & $7.69 \mathrm{hrs}$ \\
3 & $5.22 \%$ & $7.82 \mathrm{hrs}$ & $5.62 \%$ & $7.21 \mathrm{hrs}$ \\
4 & $5.81 \%$ & $7.45 \mathrm{hrs}$ & $5.12 \%$ & $7.68 \mathrm{hrs}$ \\
5 & $6.12 \%$ & $6.81 \mathrm{hrs}$ & $5.90 \%$ & $7.10 \mathrm{hrs}$ \\
6 & $5.10 \%$ & $6.65 \mathrm{hrs}$ & $6.01 \%$ & $7.32 \mathrm{hrs}$ \\
7 & $5.26 \%$ & $7.88 \mathrm{hrs}$ & $5.80 \%$ & $7.22 \mathrm{hrs}$ \\
\hline Average & $5.67 \%$ & $7.37 \mathrm{hrs}$ & $5.84 \%$ & $7.40 \mathrm{hrs}$ \\
\hline SD & 0.42 & 0.53 & 0.37 & 0.25 \\
\hline
\end{tabular}

\subsection{Alarm Response}

The Alarm Response component is responsible for rendering, based on the corresponding sleep stage, the vibrotactile stimulation pattern that must be applied to wake up the sleeper in a pleasant way. There are three types of vibrotactile stimulation patterns that we are exploring: a) simultaneous stimulation where all actuators vibrate at the same time, b) continuous stimulation which is created from an overlap of vibration from successive actuators, and c) successive stimulation where actuators vibrate separately one after the other (no two motors vibrate at the same time). Fig. 5 explains graphically the differences between the three types of stimulation patterns. As for perception of Figure 5, dark gray actuators refer to the ones activated at a time. Note that the difference between continuous and successive stimulation is that in continuous stimulation the actuation of motors must overlap over time. Successive stimulation involves non-overlapping actuation over time.

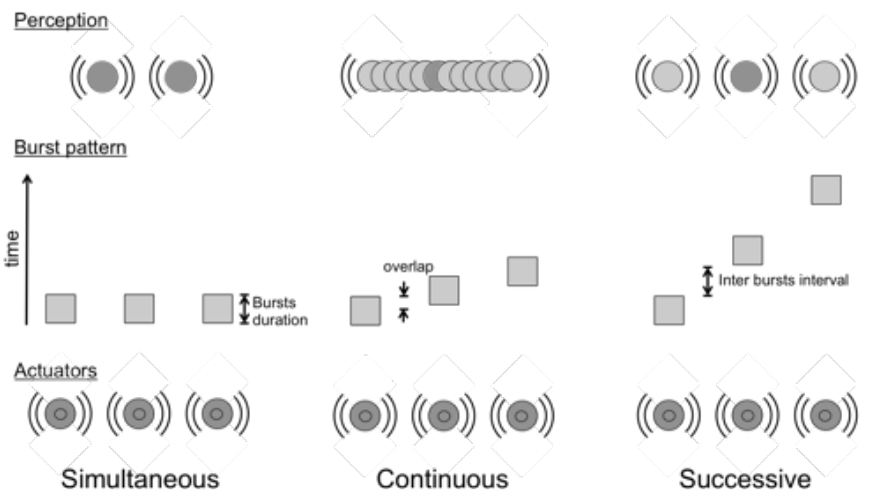

Fig. 5. The burst pattern and how this is perceived for three different modulations; simultaneous (burst duration of $100 \mathrm{~ms}$ and inter-burst interval of $300 \mathrm{~ms}$ ), continuous ( $300 \mathrm{~ms}$ burst duration and overlap of $120 \mathrm{~ms}$ ), and successive (burst duration of $150 \mathrm{~ms}$ and inter-burst duration of $150 \mathrm{~ms}$ ).

\subsection{Vibrotactile Display}

The tactile armband display device is composed of 6 vibrotactile motors that are aligned $4 \mathrm{~cm}$ apart around the wrist (Fig. 6). The selection of $4 \mathrm{~cm}$ distance between successive motors is derived from a previous perception study [38]. The device is capable of producing three types of tactile stimulation: simultaneous stimulation, successive stimulation and continuous stimulation. The three modes are explained by equation 4. A demonstration of the continuous stimulation algorithm is shown in Fig. 7. Note that the vertical axis in Fig. 7 contains the indices of the actuators that are activated in sequence.

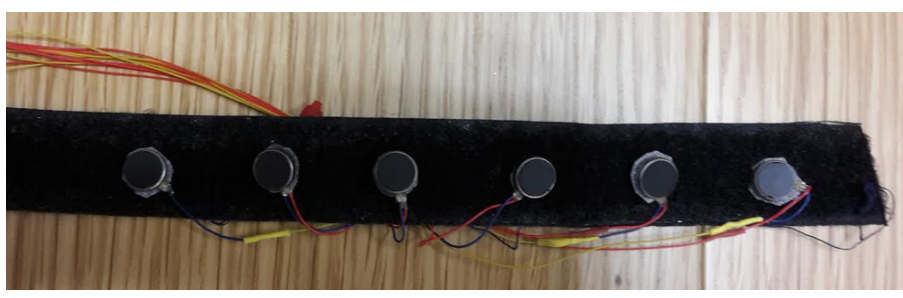

Fig. 6. Vibrotactile motors layout.

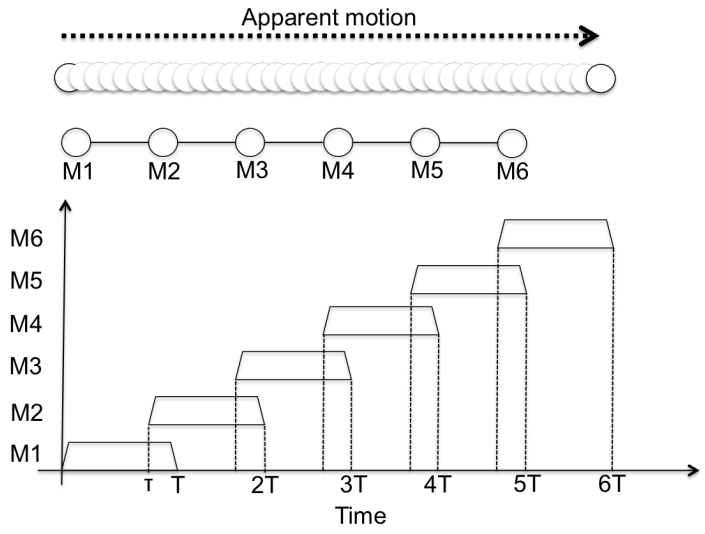

Fig. 7. Continuous tactile stimulation algorithm where $\alpha$ is between 0 and 1 (vertical axis represents corresponding motor)

$$
\tau=\alpha T, \text { where } \alpha=\left\{\begin{array}{l}
0 \text { simultaneous } \\
0 \leq \alpha \leq 1 \text { continuous } \\
\alpha \geq 1 \text { successive }
\end{array}\right.
$$

Where $\mathrm{T}$ is the burst duration, $\tau$ is the inter-burst interval and $\alpha$ is the mode factor. Simultaneous stimulation involves stimulating the motors at the same time $(\alpha=0$ or $\tau=0)$ to produce the highest sense of vibration possible where the intensity of vibration can also be controlled. Successive stimulation has one motor stimulated at a time $(\alpha>1$ or $\tau>T$ ); there is no inter-burst stimulation. Continuous stimulation is based on the funneling illusion concept [39] and produces apparent tactile motion along the actuation direction.

The stimulation intensity is controlled by adjusting the duty cycle of the Pulse Width Modulation (PWM) signal that feeds the vibrotactile actuators. Increasing the duty cycle of the PWM signal would increase the effective voltage applied to the actuator and thus the vibration intensity. The change in the intensity of vibration is linear over time, and is described by equation 5 . 


$$
I=I_{\min }+\frac{\left(I_{\max }-I_{\min }\right) \text { Gain }}{100}
$$

where $I_{\min }$ and $I_{\max }$ represent the minimum and maximum intensity respectively. The Gain variable, between 0 and 100, describes the actual intensity of vibration between $I_{\min }$ and $\left.I_{\max }\right)$.

\section{Motivating experiment: Attention To- WARDS VIBROTACTILE STIMULATION}

Although sleep and attention seem to be very different behavioral states, they are similar in that they both involve filtering out information from our awareness [40]. For example, a sleeping person is unaware of the ambient noises (unless they become very intrusive). Likewise, an awake person is able to tune into a conversation in a noisy environment by ignoring other surrounding noise (the cocktail party effect). Selective attention is the process of orienting consciousness towards, or process information from only one part of the environment with the exclusion of other parts [41]. There is evidence for attentional effects of tactile stimulation both from behavioural studies [42] and from neuronal studies [43]. Motivated by the similarity between selective attention and waking up, this experiment studies the role of vibrotactile stimulation to elicit selective attention in a multimodal scenario.

This section introduces the design, setup, and evaluation of an experiment investigating 1) the effectiveness of vibrotactile stimulation to draw the user's attention and 2) the emotional responses to different stimulation patterns. Thus we created a dual-task paradigm, where the subject is performing two tasks at once, thereby forced to divide his/her attention between the two tasks [9], [44]. The paradigm consists of a haptic detection task (where the user has to respond to a detected haptic stimulus) and a visual identification task (where the subject has to identify the correct target among different distractor stimuli).

\subsection{Experimental Setup}

The wristband providing the vibrotactile stimulus was constructed with six Pico Vibe 310-177, Precision Microdrives vibration motors with $700 \mathrm{rpm}$ frequency at a minimum intensity of $0.25 \mathrm{~g}$, and $1400 \mathrm{rpm}$ at a maximum intensity of $1.75 \mathrm{~g}$. The actuators were placed at a distance of $4 \mathrm{~cm}$ from center to center of the actuators (actuator is $10 \mathrm{~mm}$ diameter and $3.4 \mathrm{~mm}$ thickness). We created 8 patterns based on the three modulations described earlier: simultaneous, continuous and successive. A summary of the stimulation patterns is shown in Table 2. Note that the candidate patterns are derived based on a pilot study to cover all combinations of modulation attributes (three combinations for intensity, three combinations for velocity, and 2 for direction, a total of 18 combinations to start with).

Similar to other alarm clocks with increasing volume, our patterns increase in amplitude (intensity) as time passes. This was chosen partially to examine how easily the different patterns were perceived and in an attempt to make a smooth waking. Thus all of the patterns started from an intensity of $0.25 \mathrm{~g}$, which is almost not perceivable and
TABLE 2

Overview of the Different Patterns

\begin{tabular}{llll}
\hline Pattern & Modulation & Feature $^{1}$ & Attributes $^{2}$ \\
\hline 1 & Simultaneous & Int++ & $(1.25 \mathrm{~g}, 0.25 \mathrm{~g}, 10,2 \mathrm{~s}, 0,0 \mathrm{~s})$ \\
2 & Simultaneous & Int++, vel++ & $(1.25 \mathrm{~g}, 0.25 \mathrm{~g}, 10,2 \mathrm{~s}, 0,0 \mathrm{~s})$ \\
3 & Continuous & Int++ & $(1.25 \mathrm{~g}, 0.25 \mathrm{~g}, 10,2 \mathrm{~s}, 0.4,0.5 \mathrm{~s})$ \\
4 & Continuous & Int++, vel++ & $(1.25 \mathrm{~g}, 0.25 \mathrm{~g}, 10,2 \mathrm{~s}, 0.4,0.1 \mathrm{~s})$ \\
5 & Continuous & Int++, dir & $(1.25 \mathrm{~g}, 0.25 \mathrm{~g}, 10,2 \mathrm{~s}, 0.4,0.1 \mathrm{~s})$ \\
6 & Successive & Int++ & $(1.25 \mathrm{~g}, 0.25 \mathrm{~g}, 10,2 \mathrm{~s}, 3,0.1 \mathrm{~s})$ \\
7 & Successive & Int++, vel++ & $(1.25 \mathrm{~g}, 0.25 \mathrm{~g}, 10,2 \mathrm{~s}, 3,0.1 \mathrm{~s})$ \\
8 & Successive & Int++, dir & $(1.25 \mathrm{~g}, 0.25 \mathrm{~g}, 10,2 \mathrm{~s}, 3,0.1 \mathrm{~s})$ \\
\hline${ }^{1}$ Int++ is & Intensity increase, vel++ is Velocity increase, dir is Direction \\
\multicolumn{4}{c}{ change } \\
${ }^{2}$ Each cell is a set of (Imax, Imin, Gain, Duration, $\alpha$, timeStepSize)
\end{tabular}

ended after 35 to 40 seconds with an intensity of $1.25 \mathrm{~g}$, which is easily perceived. The intensity increased linearly with time.

Three of the patterns, one from each modulation type, were altered by this intensity increase: the simultaneous stimulation had a burst duration of $100 \mathrm{~ms}$ and an interburst interval of $300 \mathrm{~ms}$. The relatively short burst duration was chosen due to the effect of multiple actuators vibrating simultaneously, which intensifies the stimulation. The continuous stimulation was characterized by a burst duration of $300 \mathrm{~ms}$ and an overlap of $120 \mathrm{~ms}$ (corresponding to $40 \%$ of the burst duration). The successive stimulation comprised a $150 \mathrm{~ms}$ burst duration and an inter-burst interval of $150 \mathrm{~ms}$.

In an attempt to avoid the bias that the stimulus would become integrated in the users sleep, the next patterns were created with either increasing speed or changing direction to increase variations. Three patterns (one for each modulation) were, in addition to the intensity increase, altered by an increase in velocity. This implied a change in the interburst interval from $500 \mathrm{~ms}$ to $100 \mathrm{~ms}$ for the simultaneous modulation.

As for the continuous stimulation, this resulted in a change in burst duration from $500 \mathrm{~ms}$ to $100 \mathrm{~ms}$, while the overlap changed from $200 \mathrm{~ms}$ to $40 \mathrm{~ms}$. Furthermore, the successive stimulation increased in velocity by a change in burst duration from $250 \mathrm{~ms}$ to $50 \mathrm{~ms}$ and in inter-burst interval from $250 \mathrm{~ms}$ to $50 \mathrm{~ms}$. The last two patterns were based on continuous and successive modulation and varied in intensity and change in direction, starting from the first to the sixth actuator, and then in reversed order.

The experiment was conducted on 15 participants, all of them were students or employees of NYU Abu Dhabi; 7 were female and 8 were males. Two of the male participants were left-handed while all others were right-handed. The average age of the participants was 29.2 years ranging from 19 to 41 years. None of the participants reported any visual impairments (such as color blindness). A snapshot of this experimental setup is shown in Fig 8.

The actuators were placed on the non-dominant hand, thus if the subject was right handed, the actuators were placed around the left wrist. While the experiments took place, the subjects were listening to pink noise in order to mask any noise from the actuators.

The experiment consisted of two parts; 1) the dual-task paradigm followed by 2) subjective emotional ratings of the haptic patterns. The dual-task paradigm combined a visual 


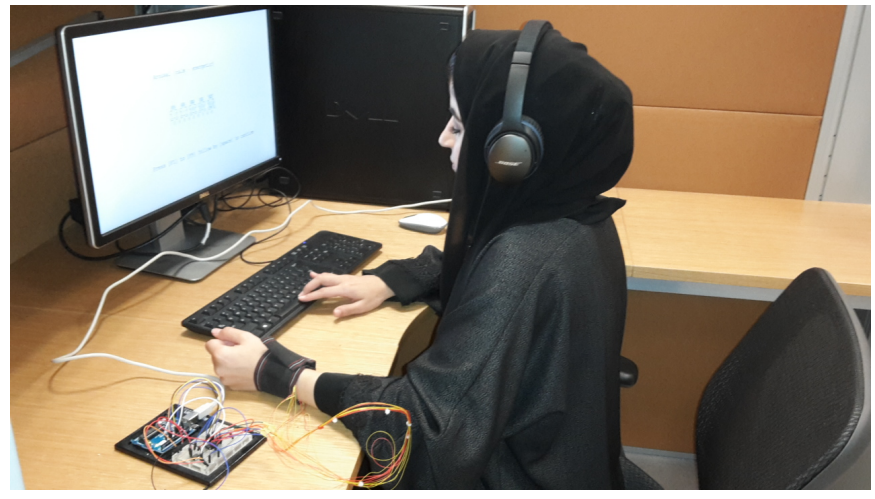

actual experiment. One subject did not complete the second part of the experiment and was therefore excluded from the analysis of the emotional ratings.

Fig. 8. The experimental setup.

(conjunction) search task [28] with a simple haptic detection task. In the visual search task, the subject searched for a target, a red plus sign " + ", among distractors that share two visual properties, color and orientation, green and red letters " $x$ " and green plus signs " + ". An example with the target present is shown in Fig. 9. If the target was identified, the user responded by pressing " $\mathrm{c}$ " for cross, but if there is no target, the user should press " $\mathrm{n}$ " for no cross. In $40 \%$ of the trials the user would also be presented with a haptic stimulus, and should respond to this by pressing space. To minimize anticipation effects of simultaneous changes of visual stimuli and appearance of haptic stimuli, the haptic stimulus would start randomly within the first 3 seconds from the beginning of the trial. Each haptic pattern started with an intensity of $0.2 \mathrm{~g}$, which is almost not perceivable. The intensity then increased over time until it reached 1.6 g. Meanwhile, the step size for increase in the intensity was the same across patterns. All 8 patterns were presented 3 times in random order. The experiment stopped as soon as the user detected all 8 haptic patterns, three times.

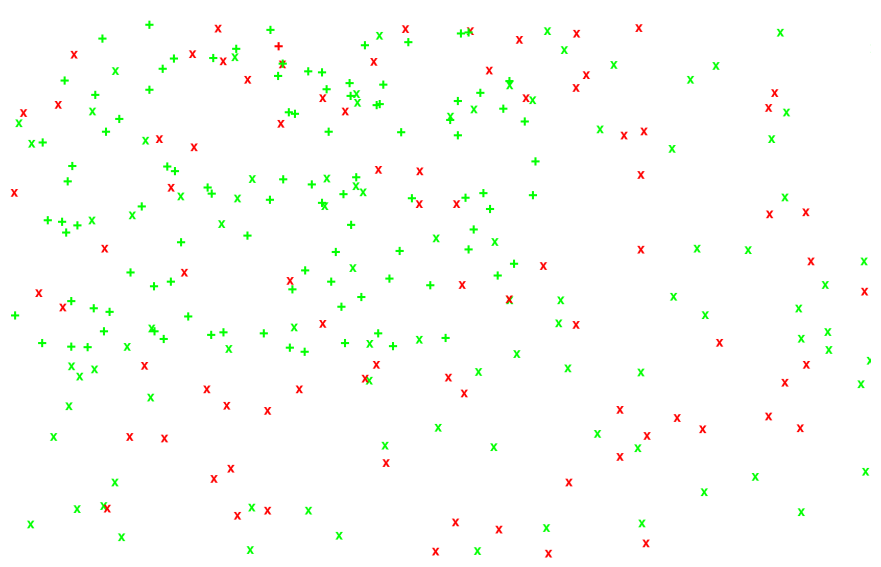

Fig. 9. The visual search task, where the subject is seeking to identify a red cross $(+)$.

In the second part of the experiment the user was presented a haptic pattern and was asked to rate this on a 9 -point valence and arousal scales (see Fig. 10). The user could repeat the stimulus by pressing " $r$ " on the keyboard. Before the experiment, the valence and arousal scales were explained to the user and three practice trials were performed, so the subject was familiarized with the scales. The stimulus presented in the practice trials was not used in the

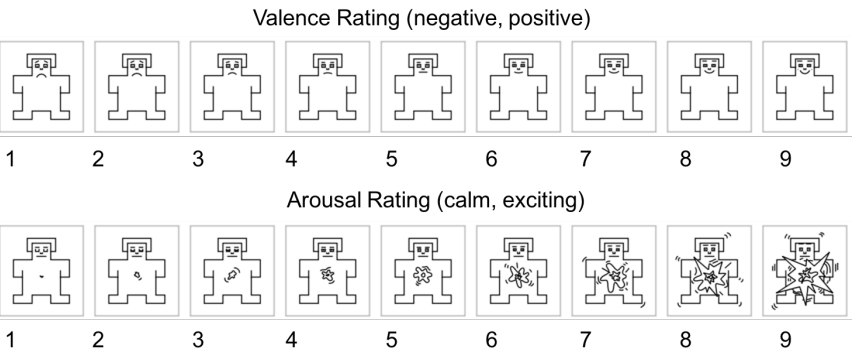

Fig. 10. The 9-point arousal/valence scales used to rate the emotional reactions [45]

\subsection{Analysis and Results}

The average response time of the three trials for each haptic pattern and for every subject was calculated. The data is plotted in a standard box plot (see Fig. 11); showing the mean as black diamonds and the median, upper and lower quartile in boxes while the whiskers represent the maximum and minimum values. The numbers on the $\mathrm{x}$-axes correspond to the pattern numbers in Table 2. The outliers are marked as circles, all of which came from the same subject. Indicating that this subject had a different perceptual threshold for tactile stimulation. A larger response time for the patterns based on the simultaneous modulation is clearly observed from Fig. 11, which is supported by the following statistical tests. Conducting one-way ANOVA analysis, both with and without outliers, showed no influence of outliers on the results. Thus we included the outliers in our analysis, and the results showed a significant effect of the patterns $\mathrm{F}(7,88)=25.61, \mathrm{p}<0.001$. To test for interaction effects two two-way ANOVA tests were conducted. One was testing two modulations (continuous and successive) with three features (intensity, intensity+velocity, intensity+direction). The other was testing three modulations (simultaneous, continuous, and successive) with two features (intensity and intensity+velocity). The first ANOVA test showed only a significant effect of modulation $\mathrm{F}(1,71)=42.249$, $\mathrm{p}<0.001$, suggesting that perception of patterns based on the continuous pattern are significantly different from patterns based on the successive pattern. The second ANOVA likewise showed a significant effect of modulation $F(2,71)=80.33$, $\mathrm{p}<0.001$. However, it also revealed an interaction effect between modulations and features $\mathrm{F}(2,71)=3.79, \mathrm{p}=0.028$. To examine the interaction effect, a permutation test with paired t-test (and 10000 reputations) and a Bonferroni correction of the significance level $(\alpha=0.05 / 15)$ is conducted. The results revealed significant difference with $\mathrm{p} \leq 0.007$ on all levels except from the tests where modulation was the same while the features were different. This indicates that the features have different effects depending on the modulation, however, not as significant as with the effect of feature.

The results of the emotional ratings on valence and arousal are shown in the box plots below, Fig. 12 and Fig. 13. The boxes represent the median, upper and lower quartile; 


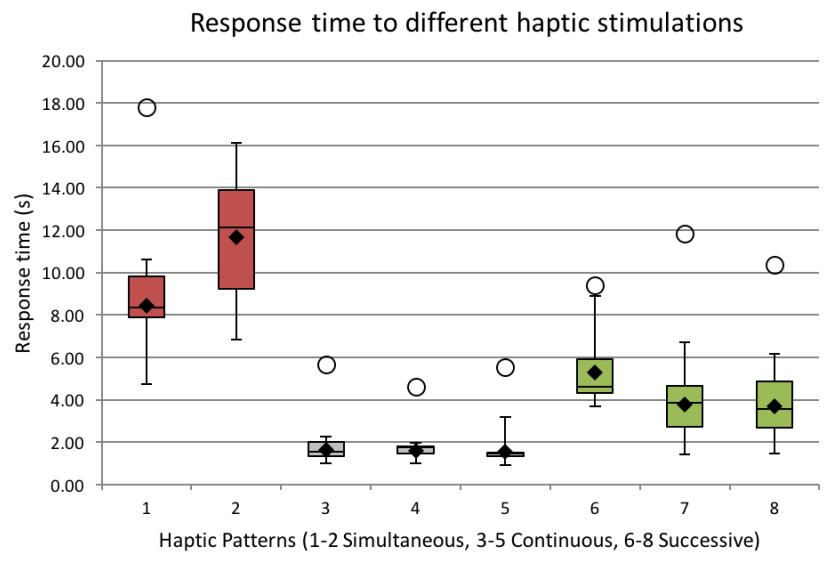

Fig. 11. The response time of 8 different haptic patterns, with diamond representing the mean and circles the outliers (consisting data from only one subject).

the black diamonds represent the mean; and the whiskers represent the maximum and minimum values. The valence data shows a large individual difference amongst subjects, which in most cases are larger compared to the arousal ratings.

Valence rating of different haptic patterns

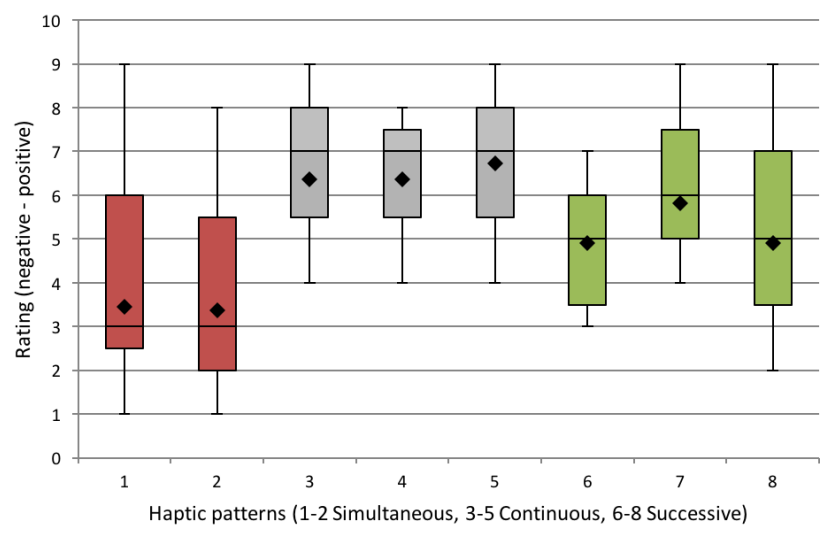

Fig. 12. The subjective valence (negative-positive) ratings of 8 different haptic stimulations, with diamond representing the mean.

By conducting a repeated measures one-way ANOVA test, we found a significant difference between the valence ratings of the patterns with $F(7,81)=7.39, p<0.001$. However, we found no significant difference for the arousal ratings. To examine the valence data further, two two-way ANOVA tests were conducted: one testing two modulations (continuous and successive) for three features (intensity, intensity+velocity, intensity+direction), the other testing the three modulations (simultaneous, continuous, and successive) with two features (intensity and intensity+velocity). The first ANOVA showed no significant effect of modulation and feature. There was no significant difference between any of the patterns based on the continuous or successive patterns. The second ANOVA showed only a significant effect of modulation $\mathrm{F}(2,65)=20.29, \mathrm{p}<0.001$. Post hoc t-test with Bonferroni

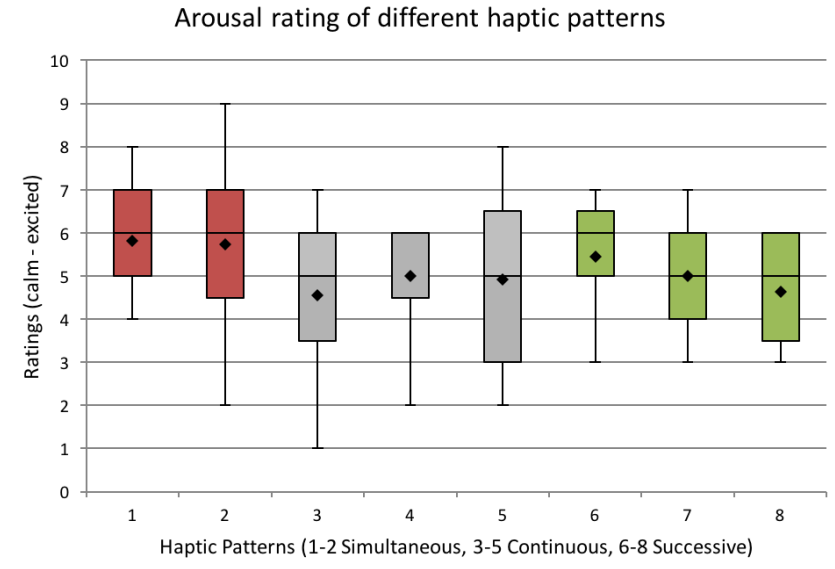

Fig. 13. The subjective arousal (calm-energetic) ratings of 8 different haptic stimuli, with diamond representing the mean.

correction $(\alpha=0.05 / 3)$ revealed patterns based on the simultaneous modulations were significantly different from those based on both continuous $(\mathrm{t}=6.16, \mathrm{p}<0.001)$ and successive $(t=3.96, p<0.001)$.

To sum up, the results on response time showed a clear effect of modulation, with the subjects responding quickest to the continuous patterns and slowest to the simultaneous ones. However, the slow reaction times for the simultaneous patterns (especially pattern 2) might be explained by the long inter-burst interval (of $500 \mathrm{~ms}$ ). It is also surprising to see how fast and similar the response times of the continuous patterns (pattern 3, 4, and 5) across subjects are. The results from the emotional ratings show greater variations amongst subjects than variations in the response times, suggesting that there might be personal preferences towards different haptic patterns. Similar to earlier reports on valence ratings, we also see higher ratings towards patterns with more continuous motions, suggesting that these are preferred over discrete motions. However, we do not see any significant effect of the arousal data and no correlations between arousal and valence data. Therefore, in order to create an effective haptic alarm, which is not only effective but also smooth and pleasant for waking up a person, our results suggest utilizing the continuous modulation.

\section{A Study of the Effects of Tactile Stimu- LATION DURING SLEEP}

After testing the functionality of the system with fully awake people in experiment 1 , the objective of experiment 2 is to investigate emotional reactions (valence and arousal) of various tactile stimulation patterns during sleep. High valence would result in pleasant awakening whereas high arousal facilitates shifting the sleeping person to the awake state. To the best of the authors knowledge, this is the first study to explore tactile stimulation during sleep.

The best-rated patterns from each modulation type are considered in this experiment: pattern 1 for simultaneous, pattern 5 for continuous, and pattern 6 for successive (based on self-reporting user study about which of the 8 patterns 
are best perceived as continuous, discrete, and simultaneous). To enhance the usability of the system during sleep, the hardware is attached to a wristband as shown in Fig. 14.

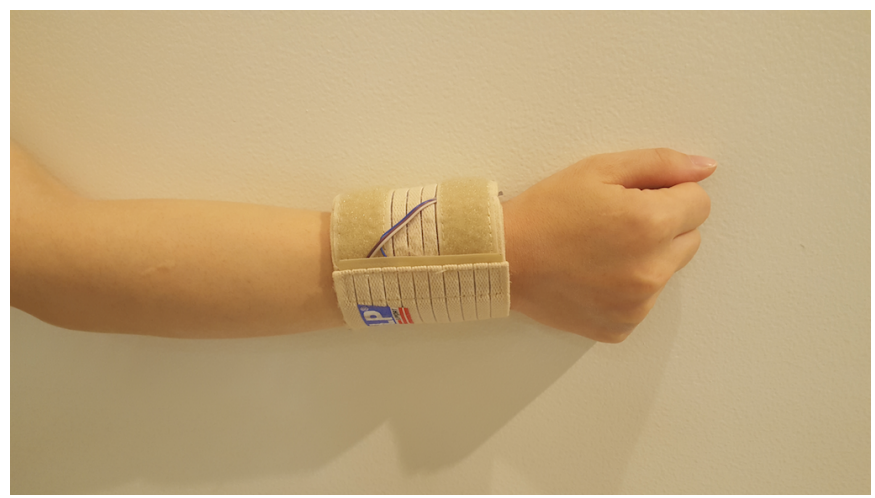

Fig. 14. The wristband device used in experiment 2 (Note that the vibrotactile motors are embedded in the wristband).

\subsection{Experimental Setup and Procedure}

Another group of ten subjects (5 males and 5 females) participated in this experiment, with age ranging from 20 to 38 years old. None of the participants reported any sleep disorders. The participants were asked to put on the wristband during regular night sleep and use the three vibration patterns as an alarm to wake up for six (6) consecutive nights, two nights per modulation pattern. For instance, participants could start for two nights with continuous stimulation, followed by two nights with successive, and finally two nights with simultaneous. The order of testing the patterns was random. The participants were instructed to complete a daily questionnaire about their quality of experience and emotional reactions to the alarm system immediately after waking up. All participants reported completing the questionnaire within 10 minutes after waking up. The questionnaire included rating valence and arousal using a 9-points Likert scale. Arousal is particularly interesting here since previous studies (such as [12]) demonstrated that high arousal may enhance waking up whereas valence measures how pleasant the experience of waking up with tactile stimulation.

\subsection{Results}

The median and distribution of valence are plotted in Fig. 15. It is clear from Fig. 15 that continuous tactile stimulation resulted in the highest scores for valence compared to successive (next best) and simultaneous (least positive). This is consistent with the results we found in experiment 1 that continuous tactile stimulation has induced the most pleasant experience for awake individuals. It seems that this is also true during sleep.

Existing research demonstrated the ability of arousal to shift in sleep states, and in particular to the awake state [12]. Therefore, the median and distribution of arousal are evaluated and plotted in Fig. 16. Continuous tactile stimulation resulted in the highest scores for arousal (slightly higher than simultaneous stimulation whereas successive stimulation was the least arousing). Furthermore, compared to previous results from experiment 1 with awake individuals,

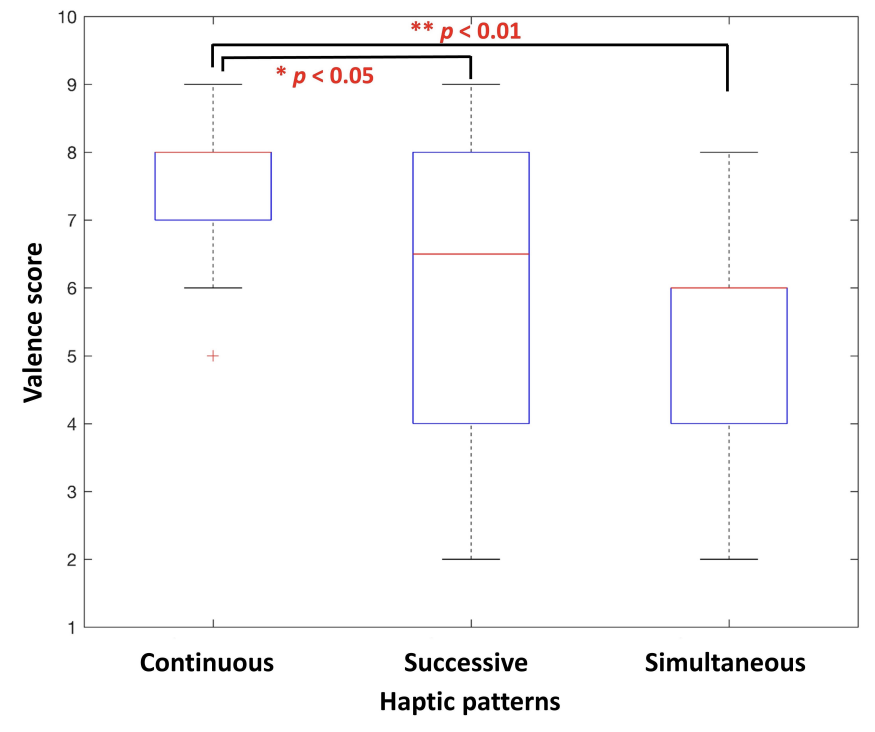

Fig. 15. The subjective valence ratings of three stimulation patterns with sleeping individuals. Repeated measures one-way ANOVA, ${ }^{* *} p<0.01,{ }^{*} p<0.05$.

it seems that continuous tactile stimulation induced slightly higher arousal during sleep and thus is arousing enough to wake up the participant. Both results of valence and arousal confirm that continuous tactile stimulation creates the most pleasant yet arousing experience to wake up sleeping individuals.

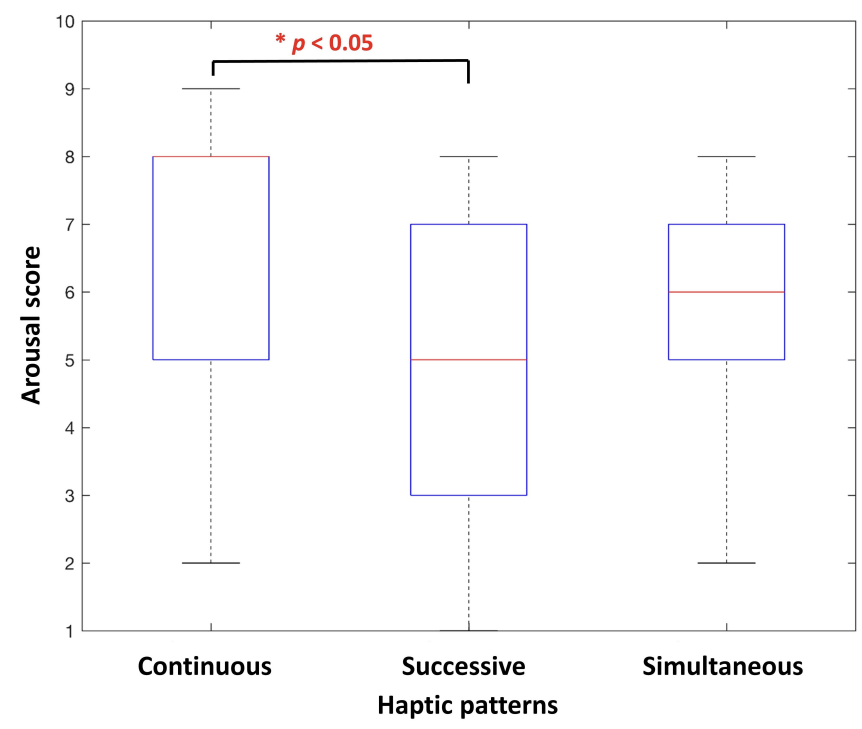

Fig. 16. The subjective arousal ratings of three stimulation patterns with sleeping individuals. Repeated measures one-way ANOVA, ${ }^{*} p<0.05$.

In order to statistically verify differences between the three modulation techniques, repeated measures one-way ANOVA analysis is conducted. There is a significant difference between the three stimulation patterns. The valence scores of continuous stimulation pattern are higher than other stimulation patterns (repeated measures one-way ANOVA, $p<0.01$ and $p<0.05)$. These results are again in agreement with previous results for both awake and sleeping individuals. In the case of arousal ratings, the scores of 
continuous stimulation pattern are higher than successive stimulation pattern (repeated measures one-way ANOVA, $p<0.05$ ).

At the end of the entire experiment, participants were asked to rank their favorite stimulation pattern. Seven participants indicated that continuous tactile stimulation is their favorite, two participant selected successive and one selected simultaneous (some favor arousal over valence or vise versa). This is probably due to individual differences between humans when it comes to tactile experiences. Therefore, individual differences must be taken into consideration when designing vibrotactile alarm systems where users may be allowed to compose/author the alarm signal that works best for them in order to create a personalized experience.

One participant suggested that on the first day, the tactile stimulation was new experience and somehow alien and thus she woke up immediately. On the second day on, her body got used to it and thus felt more comforting. The three stimulation patterns were successful to wake her up, continuous stimulation seemed to have the most pleasant effect though. Another participant reported that after a few days his body was able to adapt to the intensity of tactile stimulation and could not wake up at one night. He suggested to vary the tactile stimulation patterns (such as direction, speed, and/or intensity of tactile stimulation) over time so that users will always find them arousing in order to wake up while keeping their properties associated with positive valence. All these comments suggest that a personalized tactile stimulation pattern(s) would be more effective for individual users. All participants though agreed that they would use the system on daily basis if available as a commercial product at reasonable cost.

One explanation of preference of continuous tactile stimulation over simultaneous/successive is that continuous stimulation resembles best the experience of affective touch. Affective touch is continuous in nature, and tends to stimulate the C-fiber of the hairy skin, which in turn makes the person feel better (positive valence) [46], [47]. As for the arousing effect, affective touch is not only (neurally) represented in the somatosensory cortex but also in the limbic system [48], which plays a key role in the formation of emotions and sleep-wake transition.

\section{Conclusion And Discussion}

This paper presents a study to investigate vibrotactile stimulation as an alarm signal to effectively wake a user up a sleeping person. Experimental results demonstrate that vibrotactile stimulation induces high arousal (which helps a sleeping person wake up) and positive valence (which makes waking up a pleasant experience). More specifically, the results from experiment 1 suggest that the haptic-base response should be based on continuous modulation, since this not only is very perceivable but also rated as more positive. In Experiment 2, participants preferred the continuous stimulation pattern, with the highest scores in valance and arousal ratings.

However, as the prototype evolves, further improvements can be made to the system. The hardware could be optimized in terms of size (for example, the circuit should move to a smaller microprocessor board for even more compact assembly), and sensory data may be saved in internal non-volatile memory. A mobile device application may also be developed to provide a convenient interface to configure the system (rather than using a hand watch for now). In addition, more usability studies could be performed over longer time intervals (particularly with sleeping individuals) to further analyze the system and derive a more robust and personalized performance. One can imagine that the haptic patterns could provide the user with more information than the alarm onset. For example different patterns could indicate how close the systems alarm onset is to the users preset alarm time, thereby indicating how fast one needs to get ready. This is similar to Lylykanga's examination of patterns representing information on motion (e.g. decelerate, accelerate or keep speed constant) [28].

For future research it would be interesting to test the effectiveness of the different patterns during sleep with a larger number of participants, and over a large time interval (a few months). The tactile patterns could also be used to influence a users sleep phase, e.g. moving them from deep sleep to light sleep before waking the user. In this case it might be useful to create patterns based on simultaneous modulation, which are not that easily perceived but highly arousing. Another interesting future direction is to conduct a comprehensive study to compare tactile-based and auditory-based alarm systems.

\section{ACKNOWLEDGMENT}

The authors would like to thank Akiko Teranishi for helping in managing experiment 2 of this paper.

\section{REFERENCES}

[1] J. Kuijsten, "Adaptive Alarm Clock Using Movement Detection to Differentiate Sleep Phases," Eindhoven University of Technology, 2010.

[2] Nokia, "HappyWakeUp smart alarm clock application for Nokia mobile phones ," http://www.happywakeup.com/en/, 2010, [Online; accessed 19-April-2015].

[3] "Sleep Cycle mobile http://www.mdlabs.se/sleepcycle/.

[4] Z. Chen, M. Lin, F. Chen, N. D. Lane, G. Cardone, R. Wang, T. Li, Y. Chen, T. Choudhury, and A. T. Campbell, "Unobtrusive sleep monitoring using smartphones," in Pervasive Computing Technologies for Healthcare (PervasiveHealth), 2013 7th International Conference on. IEEE, 2013, pp. 145-152.

[5] http://www.easywake.me/.

[6] O. Krejcar and J. Jirka, "Proactive user adaptive application for pleasant wakeup," in Intelligent Information and Database Systems. Springer, 2011, pp. 472-481.

[7] K. E. Bloch, "Polysomnography: a systematic review," Technology and health care, vol. 5, no. 4, pp. 285-305, 1997.

[8] T. Bardouille, T. W. Picton, and B. Ross, "Attention modulates beta oscillations during prolonged tactile stimulation." The European Journal Of Neuroscience, vol. 31, no. 4, pp. 761 - 769, 2010.

[9] T. Kristjánsson, T. P. Thorvaldsson, and Á. Kristjánsson, "Divided multimodal attention: Sensory trace and context coding strategies in spatially congruent auditory and visual presentation," Multisensory research, vol. 27, no. 2, pp. 91-110, 2014.

[10] J. A. Anguera, J. Boccanfuso, J. L. Rintoul, O. Al-Hashimi, F. Faraji, J. Janowich, E. Kong, Y. Larraburo, C. Rolle, E. Johnston, and A. Gazzaley, "Video game training enhances cognitive control in older adults." Nature, vol. 501, no. 7465, pp. 97 - 101, 2013.

[11] D. Wang, Y. Zhang, X. Yang, G. Yang, and Y. Yang, "Force control tasks with pure haptic feedback promote short-term focused attention." IEEE Transactions on Haptics, vol. 7, no. 4, pp. 467-476, 2014 . 
[12] J. F. P. Fonseca, X. Long and R. M. Aarts, "On the impact of arousals on the performance of sleep and wake classification using actigraphy," in 35th Annual International Conference of the IEEE Engineering in Medicine and Biology Society (EMBC), vol. 4. IEEE, 2013, pp. 6760-6763.

[13] F. A. Geldard, "Adventures in tactile literacy." American Psychologist, vol. 12, no. 3, p. 115, 1957.

[14] Y. Bellik and D. Burger, "Multimodal interfaces: new solutions to the problem of computer accessibilty for the blind," in Conference Companion on Human Factors in Computing Systems. ACM, 1994, pp. 267-268.

[15] A. Israr and I. Poupyrev, "Control space of apparent haptic motion," in World Haptics Conference (WHC), 2011 IEEE. IEEE, 2011, pp. 457-462.

[16] L. M. Brown, S. A. Brewster, and H. C. Purchase, "A first investigation into the effectiveness of tactons," in Eurohaptics Conference, 2005 and Symposium on Haptic Interfaces for Virtual Environment and Teleoperator Systems, 2005. World Haptics 2005. First Joint. IEEE, 2005, pp. 167-176.

[17] D. Tsetserukou, A. Neviarouskaya, H. Prendinger, N. Kawakami, and S. Tachi, "Affective haptics in emotional communication," in 2009 3rd International Conference on Affective Computing and Intelligent Interaction and Workshops, 2009.

[18] M. Eid, A. El Issawi, and A. El Saddik, "Slingshot 3d: A synchronous haptic-audio-video game," Multimedia tools and applications, vol. 71, no. 3, pp. 1635-1649, 2014.

[19] G. Huisman, A. Darriba Frederiks, B. Van Dijk, D. Hevlen, and B. Krose, "The tasst: Tactile sleeve for social touch," in World Haptics Conference (WHC), 2013. IEEE, 2013, pp. 211-216.

[20] M. Maisto, C. Pacchierotti, F. Chinello, G. Salvietti, and D. P. Alessandro De Luca, "Evaluation of wearable haptic systems for the fingers in augmented reality applications," IEEE Transactions on Haptics, vol. PP, pp. 1-1, 2017.

[21] P. Kapur, S. Premakumar, S. A. Jax, L. J. Buxbaum, A. M. Dawson, and K. J. Kuchenbecker, "Vibrotactile feedback system for intuitive upper-limb rehabilitation," in EuroHaptics conference, 2009 and Symposium on Haptic Interfaces for Virtual Environment and Teleoperator Systems. World Haptics 2009. Third Joint. IEEE, 2009, pp. 621-622.

[22] M. M. Bradley and P. J. Lang, "Measuring emotion: the selfassessment manikin and the semantic differential," Journal of behavior therapy and experimental psychiatry, vol. 25, no. 1, pp. 49-59, 1994.

[23] M. Eid and H. A. Osman, "Affective haptics: Current research and future directions," IEEE Access, vol. 4, pp. 26-40, 2016.

[24] J. Posner, J. A. Russell, and B. S. Peterson, "The circumplex model of affect: An integrative approach to affective neuroscience, cognitive development, and psychopathology," Development and psychopathology, vol. 17, no. 03, pp. 715-734, 2005.

[25] R. W. Cholewiak and A. A. Collins, "The generation of vibrotactile patterns on a linear array: Influences of body site, time, and presentation mode," Perception $\mathcal{E}$ Psychophysics, vol. 62, no. 6, pp. $1220-1235,2000$.

[26] S. Asano, S. Okamoto, and Y. Yamada, "Vibrotactile stimulation to increase and decrease texture roughness," IEEE Transactions on Human-Machine Systems, vol. 45, pp. 393-398, 2015.

[27] H. Seifi and K. E. MacLean, "A first look at individuals' affective ratings of vibrations," in World Haptics Conference (WHC), 2013. IEEE, 2013, pp. 605-610.

[28] J. Lylykangas, V. Surakka, J. Rantala, J. Raisamo, R. Raisamo, and E. Tuulari, "Vibrotactile information for intuitive speed regulation," in Proceedings of the 23rd British HCI Group Annual Conference on People and Computers: Celebrating People and Technology. British Computer Society, 2009, pp. 112-119.

[29] J. Raisamo, R. Raisamo, and V. Surakka, "Comparison of saltation, amplitude modulation, and a hybrid method of vibrotactile stimulation," IEEE Transactions on Haptics, vol. 6, no. 4, pp. 517-521, 2013.

[30] L. Rahal, J. Cha, and A. El Saddik, "Continuous tactile perception for vibrotactile displays," in Robotic and Sensors Environments, 2009. ROSE 2009. IEEE International Workshop on. IEEE, 2009, pp. 86-91.

[31] L. Duong, M. Andargie, J.-J. Chen, N. Giakoumidis, and M. Eid, "Aegis: A biofeedback adaptive alarm system using vibrotactile feedback," in Instrumentation and Measurement Technology Conference (I2MTC) Proceedings, 2014 IEEE International. IEEE, 2014, pp. 293-298.
[32] S. Gradl, H. Leutheuser, P. Kugler, T. Biermann, S. Kreil, J. Kornhuber, M. Bergner, and B. Eskofier, "Somnography using unobtrusive motion sensors and android-based mobile phones," in Engineering in Medicine and Biology Society (EMBC), 2013 35th Annual International Conference of the IEEE. IEEE, 2013, pp. 1182-1185.

[33] M. Schmid, S. Conforto, V. Camomilla, A. Cappozzo, and T. D. Alessio, "The sensitivity of posturographic parameters to acquisition settings," Medical Engineering and Physics, vol. 24, no. 9, pp. $623-631,2002$.

[34] T. Watanabe and K. Watanabe, "Noncontact method for sleep stage estimation," Biomedical Engineering, IEEE Transactions on, vol. 51, no. 10, pp. 1735-1748, 2004.

[35] N. A. Fox, C. Heneghan, M. Gonzalez, R. B. Shouldice, and P. de Chazal, "An evaluation of a non-contact biomotion sensor with actimetry," IEEE Eng Med Biol Soc, vol. 2007, pp. 2664-2668, 2007.

[36] C. Leng, S. Wang, and H. Wang, "Learning naive bayes classifiers with incomplete data," in Artificial Intelligence and Computational Intelligence, 2009. AICI'09. International Conference on, vol. 4. IEEE, 2009, pp. 350-353.

[37] M. D. for Sleep Diagnostics and Therapy, "[47] SOMNOwatch plus EEG 6," http://somnomedics.eu/products/extended-screening/, [accessed February 10, 2015].

[38] M. Eid, G. Korres, and C. B. F. Jensen, "Soa thresholds for the perception of discrete/continuous tactile stimulation," 2015 Seventh International Workshop on Quality of Multimedia Experience (QoMEX), pp. 1-6, 2015.

[39] G. v. Bekesy, "Funneling in the nervous system and its role in loudness and sensation intensity on the skin," The Journal of the Acoustical Society of America, vol. 30, no. 5, pp. 399-412, 1958.

[40] L. Kirszenblat and B. Swinderen, "The yin and yang of sleep and attention," Trends in Neurosciences, vol. 38, pp. 776-786, 2015.

[41] N. Lavie, A. Hirst, J. de Fockert, and E. Viding, "Load theory of selective attention and cognitive control," Journal of Experimental Psychology, vol. 133, no. 3, pp. 339 - 354, 2004.

[42] P. Evans and J. Craig, "Response competition: a major source of interference in a tactile identification task," percept psychophysics, vol. 51, no. 2, pp. 199 - 206, 1992.

[43] E. Meyer, S. Ferguson, R. Zatorre, B. Alivisatos, S. Marrett, A. Evans, and A. Hakim, "Attention modulates somatosensory cerebral blood flow response to vibrotactile stimulation as measure by positron emission tomography," Annals of Neurology, vol. 29, no. 1 , pp. $440-443,1991$.

[44] A.-M. Bonnel and E. R. Haftser, "Divided attention between simultaneous auditory and visual signals," Perception $\mathcal{E}$ Psychophysics, vol. 60, no. 2, pp. 179-190, 1998.

[45] J. Morris, "Sam: The self-assessment manikin an efficient crosscultural measurement of emotional response," J. Advertising Research, vol. 35, no. 8, pp. 63-68, 1995.

[46] L. S. Löken, J. Wessberg, F. McGlone, and H. Olausson, "Coding of pleasant touch by unmyelinated afferents in humans," Nature neuroscience, vol. 12, no. 5, pp. 547-548, 2009.

[47] D. M. Lloyd, F. P. McGlone, and G. Yosipovitch, "Somatosensory pleasure circuit: from skin to brain and back," Experimental dermatology, vol. 24, no. 5, pp. 321-324, 2015.

[48] H. Olausson, Y. Lamarre, H. Backlund, C. Morin, B. Wallin, G. Starck, S. Ekholm, I. Strigo, K. Worsley, Å. B. Vallbo et al., "Unmyelinated tactile afferents signal touch and project to insular cortex," Nature neuroscience, vol. 5, no. 9, p. 900, 2002.

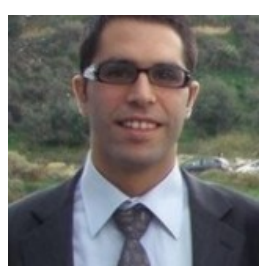

Georgios Korres studied Applied Mathematics in the School of Science and Engineering at the University of Crete. Korres was involved for several years with the development of educational software and hardware regarding educational robotics. The last three years he has also dealt with industrial automation (mainly in the field of recycling industry). His research interests focus on development of new sensors and actuators as well as the use of these in human computer interaction. 


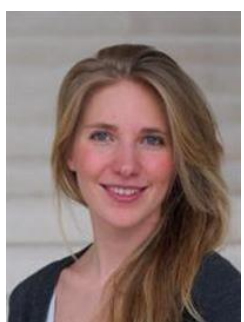

Camilla Birgitte Falk Jensen has a M.Sc. in Engineering (2012); and currently doing a Ph.D. at the Technical University of Denmark, Cognitive Systems. Publications include several conference contributions and a journal paper in International Journal of Physchophysiology. C.B.F. Jensen is doing research on mobile interaction and interface design, with focus on creating adaptive and cognitive interfaces supporting personal informatics. Her work includes interfaces for self-regulation of brainwaves using mobile equipment, with the aim of supporting the interaction between human and computer.

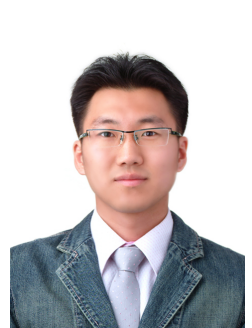

Wanjoo Park received his Ph.D. degree from the Brain and Cognitive Engineering Department, Korea University, Seoul, Republic of Korea in 2016. He had research experiments in Korea Institute of Science of Technology (KIST) from December 2008 to March 2017 as a research scientist. $\mathrm{He}$ is a Post-Doctoral Associate in the Engineering Division, New York University Abu Dhabi (NYUAD). His current research is focused on Haptics, Human-Computer Interaction, Brain-Computer Interface, Neuro-Rehabilitation and Internet Gaming Addiction. For more information, please visit the website: http://wanjoopark.wixsite.com/wanjoo

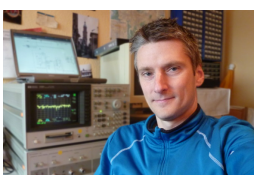

Carsten Bartsch received his M.S. (Dipl.-Ing.) in Electrical Engineering from the FAU ErlangenNrnberg in Germany. He worked for the Fraunhofer IIS in Erlangen (Germany) with focus on digital signal processing. He created software for receivers and test equipment for digital radio broadcasting. At IK4-Ikerlan in Mondragon (Spain) he developed hardware and software for a variety of industrial and European projects. His current work is in the field of human-computerinteraction and haptic technologies.

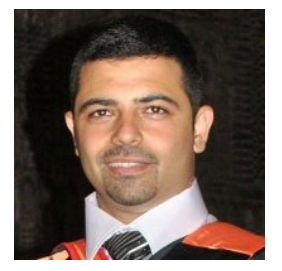

Mohamad Eid received the $\mathrm{PhD}$ in Electrical and Computer Engineering from the University of Ottawa, Canada, in 2010. He is currently an assistant professor of electrical and computer engineering in the engineering division at New York University Abu Dhabi (NYUAD). He was previously a teaching and research associate at the University of Ottawa (June 2008-April 2012). He is the co-author of the book: Haptics Technologies: Bringing Touch to Multimedia, Springers 2011, the co-chair of the 3rd International IEEE Workshop on Multimedia Services and Technologies for Ehealth (MUST-EH 2013), and has been involved in the organization of the Haptic-Audio-Visual Environment and Gaming (HAVE) workshop for the years 2007, 2008, 2009, 2010, and 2013. His academic interests include Multimedia haptics, with emphasis on affective haptics, tangible human computer interaction, and instrumentations (sensors and actuators). 